\title{
Competencias profesionales para la atención de las adicciones, en los programas de licenciatura en enfermería de la universidad autónoma de Querétaro, México.
}

\author{
Professional competencies for treatment of addictions, in undergraduate \\ programs in nursing at the Autonomous University of Queretaro, Mexico.
}

Beatriz Garza-González

Universidad Autónoma de Querétaro, México

bgarza62@gmail.com

Ma. Alejandra Hernández-Castañón

Universidad Autónoma de Querétaro, México

alehdez983@yahoo.com.mx

Aurora Zamora-Mendoza

Universidad Autónoma de Querétaro, México

auzame19@live.com

\section{Resumen}

El currículo está basado en necesidades cambiantes y en avances disciplinarios continuos, por ello, la Evaluación Curricular y el consecuente Rediseño o Reestructuración de los programas académicos son fundamentales para la pertinencia y calidad académica de los Programas Educativos. Para efectos de este documento y para dar cuenta sobre el proceso de incorporación del Modelo de Competencias al Currículo de Enfermería en el Plan 2011 del Programa de Licenciatura en Enfermería, se formuló una redimensión de su práctica profesional al incluirse, temas de geriatría y adicciones, representativos de los problemas sociales contemporáneos de la salud no sólo de nuestra entidad, sino latentes en nuestro país y en el mundo, en el entendido que como se menciona por la CICAD, que el personal de enfermería es un agentes clave para la educación sanitaria, la promoción y prevención en diferentes ámbitos (familiar, comunitario y asistencial), desde una visión innovadora. El modelo por Competencias además desarrollar en el estudiante los conocimientos, habilidades, destrezas y actitudes que le capacitan para aplicar un juicio crítico en la toma 
de decisiones, lo forma en el marco de la política pública en salud y bajo el más alto nivel de ética profesional.

Palabras clave: Adicciones, Competencia Profesional, Curriculum, Enfermería.

\section{Abstract}

The curriculum is based on changing needs and continuous disciplinary advances, therefore, Curriculum Evaluation and the consequent Redesign or Restructuring of academic programs are fundamental to the relevance and academic quality of Educational Programs. For the purposes of this document, and to report on the process of incorporation of the Competence Model to the Nursing Curriculum in the 2011 Plan of the Bachelor's Degree Program in Nursing, we formulated a new measure of his practice to include, geriatric issues and addictions, representative of contemporary health not only of our social problems, but latent in our country and the world, with the understanding that as mentioned by CICAD, the nursing staff is a key agents for health education, promotion and prevention in different fields (family, community, and health care), from an innovative vision. Competency model in addition to develop in the student the knowledge, abilities, skills, and attitudes that enable you to apply a critical judgment in decision making, is within the framework of public policy on health and under the highest level of professional ethics. Key words: Addictions, professional competence, Curriculum, Nursing.

Fecha recepción: Septiembre 2014 Fecha aceptación: Febrero 2015

\section{Introducción}

El currículo está basado en necesidades cambiantes y en avances disciplinarios que son continuos, por ello, dos de los procesos fundamentales para elevar la calidad académica y los niveles de pertinencia social de las Instituciones de Educación Superior, son la 
Evaluación Curricular ${ }^{1}$ y el consecuente Rediseño o Reestructuración de los programas académicos. La Universidad Autónoma de Querétaro preocupada siempre por la excelencia académica, ha venido apoyando las evaluaciones y reestructuraciones curriculares al interior de las facultades como estrategia para consolidar la calidad académica que ofrece a la sociedad queretana.

Del mismo modo, en los últimos años la Facultad de Enfermería ha dedicado buena parte de sus esfuerzos a la evaluación de sus programas educativos, con el objeto de contribuir al mejoramiento de una sólida formación académico-profesional que garantice la excelencia que busca la universidad. Para efectos de este documento y para dar cuenta sobre el proceso de incorporación del Modelo de Competencias al Currículo de Enfermería, retomaremos brevemente la modificación curricular que antecede al Plan 2011 del Programa de Licenciatura en Enfermería, reestructurado en el 2010, bajo estas orientaciones pedagógicas.

Las modificaciones al Plan 1997 de la licenciatura en enfermería, fueron medulares para la construcción de una visión pedagógica que facilitó las posteriores innovaciones educativas que se incorporaron al currículo. Dicha reestructuración desarrolló un plan de estudios flexible, multidisciplinario, y con una propuesta didáctica centrada en el aprendizaje. Del mismo modo se amplió la licenciatura a 10 semestres, cinco años incluyendo el Servicio Social en sus modalidades Clínico y Comunitario. También el número de asignaturas de 41 que se tenía en el plan 1997 a 68 asignaturas que estructuraba el Plan de Estudios 2004. Su fundamento se centró en la definición de ejes epistemológicos y áreas curriculares donde la formación de la autonomía intelectual en el estudiante fue la premisa de consolidación académica para la licenciatura.

\footnotetext{
${ }^{1}$ Todo proceso de modificación curricular se constituye por tres etapas bien definidas: Evaluación Curricular del programa académico vigente, para identificar su pertinencia. Trabajo colegiado para la toma de decisiones sustentadas en los resultados de la evaluación y en la investigación documental y de campo. Proceso de reestructuración y legitimación de la nueva propuesta curricular; en trabajo colectivo con académicos de la unidad educativa.
} 
En ese momento, el Plan 2004 se constituyó en una propuesta alternativa para el país, al orientar una formación innovadora más allá de la intervención profesional, incorporando otras exigencias tales como investigar los problemas sociales de salud y por ende de la práctica profesional, para una mejor comprensión de estos fenómenos.

Tomando como base este planteamiento curricular y derivado de la conjunción de esfuerzos de un grupo de académicos(as) que iniciaron en el año 2010, y como respuesta a la preocupación de las autoridades por mantener altos niveles de calidad educativa, en congruencia con la Misión, Visión y Política de Calidad de la Universidad, que a título dice:

"Participar positivamente en el desarrollo del Estado de Querétaro y del País a través de impartir educación en sus distintos tipos y modalidades del nivel medio superior y superior, procurando que la formación de profesionales corresponda a las necesidades de la sociedad; desarrollar investigación humanística y científica, tanto básica como aplicada, atendiendo primordialmente a los problemas estatales, regionales, así como a los nacionales y en relación con las condiciones del desenvolvimiento científico e histórico. Crear, preservar y difundir la cultura, así como la divulgación del conocimiento; ser agente de cambio y promoción social a través de sus tareas sustantivas” (Artículo 6º Ley Orgánica).

Se reestructuró el programa de Licenciatura en Enfermería, surgiendo el Plan 2011 que además, se deriva del Proyecto Educativo propuesto en el Plan de Desarrollo 2006-2009 de la Facultad de Enfermería como respuesta a su convocatoria a "contribuir con imaginación y determinación a fortalecer los cimientos, consolidar los avances y dirigir el nuevo rumbo de la Facultad de Enfermería para que continúe como la mejor opción en el Estado de Querétaro" (Plan de Desarrollo 2006-2009, pág. 02). Así, con dicho Plan, se pretende satisfacer las demandas crecientes de la sociedad por un mejor estado de salud y calidad de vida, renovando el compromiso social y profesional, de los y las profesionales de la salud.

El Modelo Educativo actual que subyace al Programa en mención, plantea las cuatro dimensiones que deben privilegiarse en la construcción de todo planteamiento educativo: 
Los sujetos de aprendizaje, quienes a través del desarrollo de sus estructuras cognitivas y meta cognitivas manipularán los objetos de aprendizaje, logrando con ello, apropiaciones conceptuales, procedimentales y axiológico-valorales.

La secuenciación de los contenidos: entendidos como los objetos a aprehender y de acuerdo con los principios que se derivan del aprendizaje significativo, se organizan comenzando por los elementos más generales y simples para ir introduciendo, progresivamente, los más detallados y complejos.

Los métodos y estrategias de enseñanza: dirigidas hacia la generación de ambientes de aprendizaje que permitan al estudiante adquirir el conocimiento y practicarlo en un contexto de uso lo más realista posible.

La organización social: explotando adecuadamente los efectos positivos que tiene un ambiente de intercambio en la construcción de conocimientos, desarrollar en los alumnos relaciones de cooperación y de colaboración.

Como puede observarse, estas dimensiones posibilitan que el estudiante experimente un aprendizaje significativo, que en muchas ocasiones se aproxima más a sus necesidades que los sistemas tradicionales propuestos a partir de otros modelos que hemos usado en el campo educativo.

Del mismo modo, el planteamiento curricular tiene su fundamento en el Modelo de Educación Basada en Competencias $(\mathrm{MEBC})^{2}$, integración de los "saberes" o aprendizajes teórico-conceptuales, los “saber hacer" o aprendizajes heurístico procedimentales,

\footnotetext{
${ }^{2}$ El concepto de competencia se puede ver desde tres acepciones: la que refiere a la competitividad ena ser mejor que los demás; la que se relaciona con un ámbito de responsabilidad, y la que propiamente hemos asumido en esta propuesta que se vincula con la capacidad para hacer algo, saber cómo, por qué y para qué se hace, de tal manera que pueda ser transferible. Por tanto la noción de competencia que sostiene el planteamiento que se presenta, descansa en los saberes básicos que poseerán nuestros egresados: el saber por sí mismo, como conocimiento base y explicativo que considera la comprensión; el saber hacer, como la puesta en juego de habilidades basadas en los conocimientos; el saber ser, como la parte más compleja por sus implicaciones de carácter actitudinal e incluso valoral; y el saber transferir, como la posibilidad de trascender el contexto inmediato para actuar y adaptarse a nuevas situaciones o transformarlas
} 
conjuntamente con los "saber ser" o aprendizajes axiológico-actitudinales. Esto es, la convergencia de comportamientos sociales, afectivos y habilidades cognoscitivas, psicológicas, sensoriales y motoras, que permiten llevar a cabo una actividad o una tarea específica (Argudín, 2001).

En este sentido, se exponen las bases epistemológicas y pedagógicas que sustentan el Programa de Licenciatura en Enfermería Plan 2011 y que promueven la incorporación del MEBC:

Se orienta a la formación científica y humanística. El programa amalgama una educación humanística con el rigor científico, en donde la integración de diversas disciplinas aportan elementos teórico-metodológicos, para formar estudiantes en habilidades intelectuales y manuales, a modo de cultivar la autonomía intelectual, imperativo fundante de esta propuesta, que se exprese en estudiantes formados con conciencia histórica, teórica y crítica, como medios esenciales de su pensar, hacer y actuar y enfrentar la realidad y su formación como persona vinculada a un contexto económico, político y social.

Las mismas disciplinas propuestas, constituyen áreas curriculares que se integran entre sí y superan a los planes anteriores en su profundidad y riqueza; la sociohistórica, la psicosocial, la técnico-profesional y la de investigación, en donde la educación moral también es educación política de la conciencia, de la ciencia y la tecnología; en su conjunto amplían, el interés, compromiso e intervención a favor de anticiparse a plantear retos alternativos a los problemas de salud-enfermedad.

Potencia el aprendizaje. Su concepción y aplicación pedagógico-didáctica, se centra fundamentalmente en el dejar aprender al estudiante, a través de procesos que promueven el pensamiento dilemático, analítico y problematizador; tanto en la realidad de la enfermería y su entorno social, como de las disciplinas que la explican, a partir de experiencias personales y colectivas para problematizar, reconstruir y crear, lo que encamina al proceso de organización del pensamiento y la razón. Para la enseñanza tanto de la teoría como para el desarrollo de habilidades, el Plan de Estudios plantea la perspectiva didáctico-pedagógica desde el constructivismo pedagógico. 
Es integrador. En el proceso formativo, el Mapa Curricular amalgama los ejes de formación (teórico-profesionalizante, técnico-metodológico y transdisciplinar), que a su vez articulan las funciones que desempeña el profesional de enfermería, con las áreas curriculares: Biológica, Enfermería, Metodológica-instrumental, Social comunitaria y de Psicología y desarrollo humano, constituyendo, una integración de saberes con sentido y especificidad para la profesión.

Es abierto y sistemático. Porque el proceso de formación docente y la evaluación curricular se asumen como permanentes y de retroalimentación mutua, acoplando la discusión en trabajo colegiado para resolver problemas e investigar la práctica docente cotidiana, con la actualización didáctico-pedagógica y disciplinar en las áreas que constituyen el programa, facilitando a la vez, la incorporación ágil y articulada de los nuevos conocimientos. Asimismo, abierto, en sentido de flexibilidad curricular que favorece la movilidad efectiva de los estudiantes mediante estrategias de no seriación de contenidos y materias optativas.

Es innovador. Como experiencia formativa, en el ámbito de licenciatura, el currículo se innova, al menos en los tres grandes rubros (las dimensiones del Modelo educativo, el enfoques por competencia y las áreas de integración curricular) que se ven fortalecidos.

Esta panorámica, exige la necesidad de transformar la formación de los licenciados(as) en enfermería que coadyuven a mejorar los niveles de salud en el ámbito preventivo y curativo, con sólida formación científica y humanística basada en el aprendizaje significativo que les permita evaluar, investigar e intervenir en sus diferentes ámbitos de acción. En donde la investigación se constituya uno de los soportes fundamentales como medio para acercar la teoría de enfermería a nuestra realidad social, así como incluyendo nuevos saberes y prácticas necesarios para actuar en su entorno local y regional. Un nuevo tipo de profesional comprometido con su realidad y creativo para dilucidar y abrir otras posibilidades del ejercicio profesional.

Importancia de la inclusión de contenido de drogas en los currícula de Licenciatura en Enfermería 
La explosión de las adicciones a nivel mundial es considerada hoy, no sólo como resultado de las variadas dificultades que enfrenta el individuo o grupos en la vida contemporánea, sino también de la ausencia sistemática de la educación sanitaria. Como ya se ha mencionado, el incremento en el consumo tanto de drogas legales como ilegales así como las consecuencias negativas asociadas a las adicciones que afectan la esfera física, psicológica (cognitiva y afectiva) y espiritual en la vida de una persona, aunado a situaciones de índole socioeconómica, es una de las grandes preocupaciones del sector salud.

En los países de América, al igual que el reto del mundo, ha sido planteado por la Organización de los Estados Americanos (OEA), indicando que es alarmante el aumento de las adicciones en diversos grupos de población, en los que el grupo de mayor riesgo se encuentra en los adolescentes. Respecto a esta problemática, se emprende la Alianza Hemisférica contra la Droga, emprendida por la OEA a través de la Comisión Interamericana para el Control del Uso Indebido de Drogas (CICAD), propone estrategias tendientes a reducir este fenómeno en donde se considera a las enfermeras agentes claves de intervención para brindar una mayor educción sanitaria, investigar y proponer sobre este problema.

En México, y particularmente en nuestro estado, es preocupante el incremento en el consumo de drogas legales e ilegales así como las consecuencias negativas asociadas a las adicciones. La Encuesta Nacional de Adicciones, (INPRFM, 2011) reveló que las mujeres de Querétaro en edades entre18 a 65 años, tienen la prevalencia más alta del país en consumo de alcohol, además de que se incrementó la oportunidad de los adolescentes para el uso de las drogas, para lo cual se requiere de acciones conjuntas con los Consejos Estatal y Municipal a través de programas preventivos y curativos, así como la certificación y regulación de centros y clínicas contra las adicciones. Éste es uno de los ámbitos en los que la enfermería hace presencia, particularmente nuestra facultad, a través del Programa de Enfermería y Salud Interactiva (ENSAIN) y de su división Prevención de Adicciones (PA), cuyas actividades de extensión en salud preventivo-comunitaria se realizan mediante convenios interinstitucionales y programas intrainstitucionales. 
Finalmente, los retos y estrategias del sector salud se orientan a mejorar las condiciones de salud de la población del estado mediante el acceso universal a los servicios de salud, el fortalecimiento de programas preventivos y atención con un enfoque humanista. Las estrategias a seguir para el logro de objetivos que particularmente se orientan a la problemática de las adicciones, planteadas por la Secretaria de Salud en el estado, son:

Reforzamiento de la protección y prevención de la salud basado en la estrategia del Paquete Básico Garantizado de Prevención y Promoción para una Mejor Salud.

Prevención de las adicciones

Fortalecimiento del capital humano, la investigación y la enseñanza en salud (SESEQ, 2010)

Propósitos que se articulan a las directrices de las organizaciones internacionales que operan para mejorar la salud de las poblaciones: La Organización Mundial de la Salud, (OMS), instancia especializada de la Organización de las Naciones Unidas que realiza actividades de investigación, coordinación y distribución de información a todos los países del mundo, la Organización Panamericana de Salud (OPS), La Organización para la Cooperación y el Desarrollo Económico (OCDE), La Organización de Derechos Humanos de la ONU, la Comisión Interamericana para el Control y Abuso de Drogas (CICAD) de la misma dependencia, así como los Sistemas de Salud y la Secretarías de Salud.

Derivado de lo anterior, y bajo la concepción de que un elemento clave para evaluar la pertinencia de un programa educativo es su vinculación con la problemática social, se formuló una redimensión de la práctica profesional de enfermería, al incluirse en la discusión para la construcción del currículum, temas de geriatría y adicciones, representativos de los problemas sociales contemporáneos de la salud no sólo de nuestra entidad, sino latentes en nuestro país y en el mundo.

Como ya se mencionó, la CICAD, afirma que el personal de enfermería son agentes clave para intervenir en los grandes problemas socio-sanitarios de nuestro país. Por lo anterior, dentro de los contenidos del programa se pretende el estudio de las adicciones revisadas en extensión, con el propósito de que nuestros egresados lleguen a la comprensión de este fenómeno en toda su integralidad (político, económico, cultural, biológico y psicológico), 
con dominios suficientes de conocimiento para problematizarlo en sus múltiples manifestaciones. Se busca, por tanto, considerar este problema de salud como objeto de investigación e intervención sistemática por este profesional de la salud, haciendo énfasis en un sentido más sociólogo de intervención, es decir, en la educación sanitaria, promoción y prevención en diferentes ámbitos del entorno familiar, escolar, laboral, comunitario, regional o municipal.

De este modo, el perfil profesional de la Licenciatura en Enfermería Plan 2011, se orientó al desarrollo de competencias profesionales que le permitan al egresado, gestionar los servicios de salud, trabajar profesionalmente en programas educativos en enfermería desde una visión innovadora, así como desarrollarse en el ámbito asistencial; además desarrollar en él, conocimientos, habilidades, destrezas y actitudes que le capaciten para aplicar un juicio crítico en la toma de decisiones, distinguiéndose por su aportación y liderazgo dentro del equipo de salud, en el marco de la política pública de salud y bajo el más alto nivel de ética profesional.

Dichas tareas se especifican en los campos de formación desde la visión funcional, de ahí se desprende que el egresado (a), será capaz de realizar las siguientes funciones profesionales:

-Promotor(a) de la salud: El profesional desarrollará esta función en los ambientes domiciliario, vecinal, escolar, clínico, laboral, comunitario o regional. Asume los roles de consejero (a) de salud, promotor (a) y asesor (a) sanitario para el autocuidado, donde las personas y población conoce las medidas necesarias para el fomento a la salud y a la prevención de enfermedades y por tanto asuman la actitud y afronte la voluntad de mantenerse sanos.

-Proveedor(a) de cuidados: Contribuirá en la atención integral biopsicosocial que se proporciona a la persona para satisfacer las necesidades básicas de la vida cotidiana y a las alteraciones de las funciones corporales a través de cuidados secundarios y terciarios en instituciones de salud, así también realizará intervenciones de atención ambulatoria, primaria, secundaria y de rehabilitación, en donde la gerontología y las adicciones 
adquieren especial significado como campos profesionales emergentes de intervención disciplinario o interdisciplinario.

-Educador(a) para la salud: Diseña, interviene y evalúa programas de prevención, conservación y recuperación de la salud, con base en los diagnósticos de enfermería individual, familiar o comunitario así como los diagnósticos epidemiológicos. Así también enseñanza, actualización y evaluación al personal de las instituciones de salud y de personas de la comunidad que ejecutan el rol de cuidadores de la salud.

-Gestor(a) de servicios: Realizará tareas de planeación, diseño, coordinación y evaluación de la gestión de la atención y servicios de enfermería, así como de programas de salud en general.

-Investigador(a): Realizará diseños y elaboración de investigación que aborden problemas que la profesión plantea, desde lo epistemológico y la práctica cotidiana, hasta el requerimiento de soluciones o propuestas alternativas (Fundamentos Socioacadémicos del Programa de Licenciatura en Enfermería, Plan 2011).

\section{Estrategias de implementación del curriculum para el desarrollo de competencias}

El fenómeno de las adicciones es un problema de salud pública que ha crecido en los últimos veinte años de manera exponencial, confrontando valores familiares y la efectividad de la educación, representa un reto de gran importancia en cuanto a recursos e infraestructura para la atención, con especial énfasis en la reducción de la demanda y las acciones de promoción y prevención de la salud. Por ello, es importante que los programas de prevención incluyan a la familia y que se refuercen los programas encaminados a lograr que las personas con problemas en el consumo de alcohol se acerquen a tratamientos.

Los adolescentes y jóvenes de hoy están más expuestos a la oportunidad de consumir drogas que las generaciones anteriores; por ello, actualmente el uso y abuso de éstas es en mayor proporción. De ahí la necesidad de orientar la prevención fortaleciéndolos frente al 
riesgo. Se trata, por tanto, de un fenómeno que afecta principalmente a los grupos más jóvenes. Los perfiles epidemiológicos sobre todo en la población referida, están fuertemente influidos por los factores psicosociales que intervienen en la adopción de estilos de vida no saludables. Muchos de los problemas de salud de los adolescentes y jóvenes, incluidos los problemas de morbilidad y mortalidad excesiva, son generados por conductas de riesgo evitables, tales como accidentes, actos violentos y enfermedades de transmisión sexual, asociados a las adicciones.

A pesar de que, como ya se mencionó, la problemática de adicciones responde fuertemente a estereotipos y roles masculinos, el consumo de esas sustancias por parte de las mujeres muestra una tendencia creciente, por lo que es indispensable profundizar el conocimiento así como los medios de prevenirla y atenderla considerando las diferencias entre mujeres y hombres.

La participación del profesional de enfermería en la promoción de la salud y la prevención de la enfermedad, utilizando como herramienta la vigilancia epidemiológica o el monitoreo estratégico que brinda la epidemiología social, debe garantizar una visión integrada de los componentes en la promoción y prevención de las adicciones que, desde la profesión, se incorporan en el análisis y solución de una problemática como ésta. Así, en la utilización del proceso de enfermería al estudiar la etiología de la dependencia de drogas es importante tener en cuenta los factores no farmacológicos, como la disponibilidad, las modas, la presión del grupo y el aprendizaje previo con sustancias toleradas socialmente pero no exentas de riesgo. Además, considerar que la vulnerabilidad a la farmacodependencia está también relacionada con algunas características del individuo habiéndose incluso reconocido en algunos casos la existencia de factores hereditarios.

El Proceso de Enfermería es traducido fundamentalmente de la metodología científica y ha sido legitimado por la enfermería como el marco conceptual y metodológico por excelencia, para la intervención en el cuidado del enfermo. Es el método sistemático para administrar el cuidado de enfermería, en donde la deliberación y reflexión técnica y de deliberación y reflexión práctica son constitutivas para valorar, identificar alteraciones de 
salud o estado de equilibrio, planear, intervenir y evaluar las necesidades humano-sociales y biológicas de los individuos.

En esa perspectiva, es incorporado como uno de los ejes integradores del currículo para la formación disciplinar y metodológica de los estudiantes, como uno de los instrumentos básicos (conjuntamente con otros métodos: científico, clínicos, administrativo, epidemiológico) que les posibilite para problematizar la naturaleza y la justificación de los fines y objetivos prácticos, desarrollando la capacidad de observación y análisis vinculadas en lo manual e intelectual.

Por otro lado, en el origen y el mantenimiento de una farmacodependencia intervienen numerosas variables, por esta razón el estudio y entendimiento requiere de un enfoque multidisciplinario. Algunas variables que son dignas de destacar son las propiedades farmacológicas de las sustancias, los condicionantes fisiológicos y psicológicos del consumidor, así como diversos factores sociales y ambientales. De tal forma que les permita conocer y dar respuesta a problemáticas derivadas de su consumo en las diferentes etapas del ciclo vital (niñez, adolescencia, adultez y vejez), en los ámbitos comunitario y clínico utilizando el método Proceso de Enfermería. El énfasis, seguirá siendo la preeminencia de la educación sanitaria y estrategias de intervención para contribuir al proceso de reinserción social, de manera interdisciplinaria y en cooperación interinstitucional. Enfatizando en la génesis y el mantenimiento de una adicción intervienen numerosas variables, por esta razón el estudio de este fenómeno requiere de un enfoque multidisciplinario.

El proceso de enfermería es un método ordenado y sistemático que sirve para obtener información e identificar los problemas del individuo, la familia y la comunidad (Alfaro 2007), con el fin de planear, ejecutar y evaluar el cuidado de enfermería, que se ocupa de mantener, promocionar y proteger la salud, cuidar al enfermo y ayudar a la rehabilitación. Por tanto, es la aplicación del método científico en el quehacer de enfermería, que delimita su campo de acción específico y denota el desarrollo de numerosas acciones que van más allá del cumplimiento de una prescripción médica (Rodríguez 2000). Por ello, la aplicación de éste sobre el estudio de la etiología del uso y abuso de drogas, así como la dependencia, 
considera importante tomar en cuenta los factores no farmacológicos, los condicionantes fisiológicos y psicológicos del consumidor, así como diversos factores sociales y ambientales que pueden llevar a la disponibilidad, las modas, la presión del grupo y el aprendizaje previo con sustancias toleradas socialmente, pero no exentas de riesgo; además, la vulnerabilidad a la farmacodependencia está relacionada también con factores de socioeconómicos y culturales.

Como hemos venido mencionando, el estudio de la Enfermería se basa en el conocimiento de la persona desde una perspectiva integral que la interrelaciona con su entorno sociocultural y ambiental (estructura del cuerpo humano, desarrollo psicológico y sociedad y apoyo social), con los conocimientos de salud hospitalaria y comunitaria y con la adquisición de las habilidades y los fundamentos propios de la profesión. Desde esta visión holística e integral, se plantea una formación profesional que además de promover el desarrollo de ciertos atributos (habilidades, conocimientos, actitudes, aptitudes y valores), considere la ocurrencia de varias tareas (acciones intencionales) que suceden simultáneamente dentro del contexto en el cual tiene lugar la acción, y a la vez permita que algunos de estos actos intencionales sean generalizables (Gonczi, 1996).

El objetivo curricular que determina el perfil de egreso del programa académico, explicita los saberes, saberes hacer y ser, de los futuros egresados, mismos que, en su aplicación directa en situaciones profesionales se convierten en las competencias ${ }^{3}$ a desarrollar en los y las estudiantes de la Licenciatura en Enfermería. (Figura 1).

\footnotetext{
${ }^{3}$ En este sentido, el estudio de la Enfermería se basa en el conocimiento de la persona desde una perspectiva integral que la interrelaciona con su entorno sociocultural y ambiental (estructura del cuerpo humano, desarrollo psicológico y sociedad y apoyo social), con los conocimientos de salud comunitaria, la adquisición de habilidades y los fundamentos propios de la profesión. Desde esta visión holística e integral se plantea una formación profesional que además de promover el desarrollo de ciertos atributos (habilidades, conocimientos, actitudes, aptitudes y valores), considere la ocurrencia de varias tareas (acciones intencionales) que suceden simultáneamente dentro del contexto (y la cultura del lugar de trabajo) en el cual tiene lugar la acción; y a la vez permita que algunos de estos actos intencionales sean generalizables" (Gonczi, 1996).
} 


\section{Figure 1. Formation of competition}

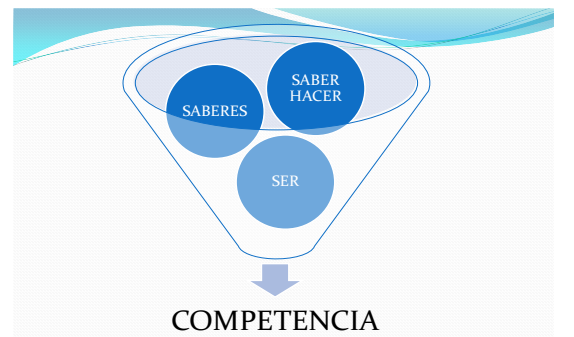

Así, para el logro del objetivo curricular propuesto y de acuerdo al Modelo educativo que subyace a la educación en nuestro país, este planteamiento curricular se estructura bajo el Modelo de Competencias, siendo las Básicas aquellas que dan sustento a la formación y sirven de pilar para el tránsito y desarrollo de las competencias Disciplinares que, en su conjunto con las primeras, constituyen las Competencias Profesionales a desarrollar por el estudiante de la Licenciatura en enfermería, Plan 2011.

A partir de lo anterior, se pretende que al finalizar sus estudios los egresados del programa posean los conocimientos, las aptitudes, las habilidades y las actitudes competencias profesionales- que le permitan comportarse y actuar como un profesional de la enfermería. Convirtiéndose así en agentes de cambio capaces de analizar y promover las modificaciones, a nivel individual y colectivo, que permitan una constante evolución conceptual, científica y tecnológica de la Enfermería.

Es importante destacar que los rasgos o características enunciadas en el perfil profesional, no corresponden de manera exclusiva a una asignatura o actividad específica: algunos, como el dominio de ciertos contenidos y técnicas, se identifican primordialmente con espacios curriculares delimitados en el plan de estudios; otros, como la consolidación de las habilidades intelectuales o la formación valoral, corresponden a los estilos y las prácticas educativas que se promoverán en el conjunto de los cursos. 
De este modo, el planteamiento curricular del Plan 2011, está organizado a partir de tres ejes de formación:

Eje I Teórico - Profesionalizante en donde se promueven lenguajes indispensables para la formación profesional del licenciado en Enfermería. A través de éste se asegura el dominio de las bases disciplinares y su fundamento se encuentra en el Proceso de Enfermería.

Las áreas curriculares y asignaturas que integran este eje de formación son:

- BIOLÓGICA (BIO01/10): Bioquímica, Anatomofisiología I y II, Ecología y Salud, Fisiopatología I y II, Farmacología, Gerontología y Geriatría, Pediatría y GinecoObsetricia y Perinatología.

- ENFERMERÍA (ENF01/26): Introducción a la Enfermería, Proceso de Enfermería, Enfermería Fundamental, Cuidado del Adulto, Cuidado Quirúrgico, Cuidado del Adulto mayor, Cuidado del trinomio madre-padre-hijo, Cuidado del niño y el adolescente, Cuidado de la salud comunitaria I y II, Salud y seguridad en el trabajo, Cuidado alternativo, Seminario de actualización disciplinar, Laboratorios, Prácticas y Servicio Social.

El Eje II Metodológico contribuye con su mediación instrumental a la articulación de todos los saberes en la resolución de problemas dentro del contexto profesional, laboral y social. Las áreas curriculares y asignaturas que integran este eje de formación son:

Desde esta perspectiva, la inclusión de los contenidos explícitos sobre adicciones, se ha vinculado directamente con el eje de formación transdisciplinar mismo que agrupa materias importantes para el ejercicio de la profesión. Las áreas curriculares y asignaturas que integran este eje de formación son:

- SOCIAL-COMUNITARIA (SC01/07): Estado Sociedad y salud, Activación Física I y II, Activación Física y Rehabilitación, Cuidado de la Salud Pública, Adicciones I y II. 
A continuación se presentan las competencias establecidas en el Plan de Estudios 2011, que promueven el abordaje profesional en el ámbito de las adicciones, así como las asignaturas que las apuntalan desde sus contenidos:

\begin{tabular}{|c|c|}
\hline COMPETENCIAS & ASIGNATURAS \\
\hline $\begin{array}{l}\text { En el transcurso de su aprendizaje desarrolla la comprensión y el compromiso de una } \\
\text { participación interdisciplinaria, para la instrumentación de programas de salud } \\
\text { dirigidos a la población e intervenir en acciones de salud en los diferentes niveles de } \\
\text { atención con base en el perfil epidemiológico predominante. Bajo una postura } \\
\text { consciente de apertura a la búsqueda y construcción de nuevos conocimientos en su } \\
\text { vida profesional y personal. } \\
\text { Revaloriza la práctica profesional de la enfermera (o) en el espacio de intervención } \\
\text { comunitaria, sobre la base de un trayecto formativo, enfatizando en forjar una } \\
\text { conciencia social, analizar, problematizar y aprehender la realidad y resolver la } \\
\text { problemática de salud ahí presentes. }\end{array}$ & $\begin{array}{l}\text { Estado, sociedad y } \\
\text { salud } \\
\text { Cuidado de la salud } \\
\text { pública } \\
\text { Cuidado de la salud } \\
\text { comunitaria I y II }\end{array}$ \\
\hline $\begin{array}{l}\text { Genera propuestas para la atención de enfermería tendientes a cuestionar patrones de } \\
\text { conducta obsoletos de la enfermera, a modificar la conducta y actitud de la población } \\
\text { ante su proceso salud enfermedad y promoviendo la creación de propuestas que } \\
\text { garanticen la calidad de la atención de enfermería, en la realidad de una práctica } \\
\text { profesional dinámica y compleja. } \\
\text { Analiza y toma decisiones de acuerdo a las circunstancias prevalecientes en la } \\
\text { problemática de salud o enfermedad de los individuos, ajustándose a los hábitos y } \\
\text { creencias de las personas, en el contexto de la conservación de la salud o curación y } \\
\text { rehabilitación de esta. } \\
\text { Transforma paulatinamente la práctica profesional en los campos dominantes y } \\
\text { emergentes enfrentando una sociedad dinámica y compleja, utilizando como medios } \\
\text { a la investigación, la gestión, el liderazgo y la intervención; a través de la vinculación } \\
\text { de conocimientos, saberes, habilidades, técnico profesionales y técnico cognitivas, } \\
\text { actitudes. }\end{array}$ & Adicciones I y II \\
\hline $\begin{array}{l}\text { Disposición para aplicar los diversos métodos: clínico, epidemiológico, científico, en } \\
\text { la investigación de resolución de problemas de los procesos de interacción } \\
\underline{\text { comunicativa, proceso de atención de enfermería, en el campo de acción enfermero. }}\end{array}$ & $\begin{array}{l}\text { Cuidado de la salud } \\
\text { comunitaria I y II } \\
\text { Cuidado de la salud } \\
\text { pública }\end{array}$ \\
\hline $\begin{array}{l}\text { Cultiva y promueve valores, derechos humanos, universales y los valores culturales } \\
\text { regionales y nacionales, en el ejercicio de la enfermería. }\end{array}$ & $\begin{array}{l}\text { Estado sociedad y } \\
\text { salud }\end{array}$ \\
\hline $\begin{array}{l}\text { Fomenta una conciencia histórica y crítica sobre los problemas que el contexto } \\
\text { histórico plantea a la profesión. }\end{array}$ & $\begin{array}{l}\text { Estado Sociedad y } \\
\text { salud } \\
\text { Adicciones I y II }\end{array}$ \\
\hline $\begin{array}{l}\text { Contextualiza, interpreta y confronta desde la experiencia y la conceptualización, los } \\
\text { fenómenos particularmente problemáticos de la práctica profesional en su } \\
\text { vinculación con el proceso de salud-enfermedad, profundizando en la especificidad } \\
\text { de la estructura social y antropológica de esa vinculación. }\end{array}$ & $\begin{array}{l}\text { Estado sociedad y } \\
\text { salud } \\
\text { Cuidado de la salud } \\
\text { pública }\end{array}$ \\
\hline $\begin{array}{l}\text { Aplica cuidado integral de enfermería, reconociéndose como parte integrante del } \\
\text { equipo multidisciplinario, en la atención de las necesidades del individuo o } \\
\text { colectividad sana o enferma, reconociendo los ámbitos y niveles de competencia y } \\
\text { perspectivas de cada uno. }\end{array}$ & $\begin{array}{l}\text { Cuidado de la salud } \\
\text { comunitaria I y II }\end{array}$ \\
\hline $\begin{array}{l}\text { Sustenta la búsqueda y generación del conocimiento empírico y conceptual, } \\
\text { desarrollando progresivamente habilidades conceptuales - metodológicas, integrando } \\
\text { y confrontando contenidos y discursos asimilados en su proceso }\end{array}$ & $\begin{array}{l}\text { Estado sociedad y } \\
\text { salud } \\
\text { Cuidado de la salud }\end{array}$ \\
\hline
\end{tabular}




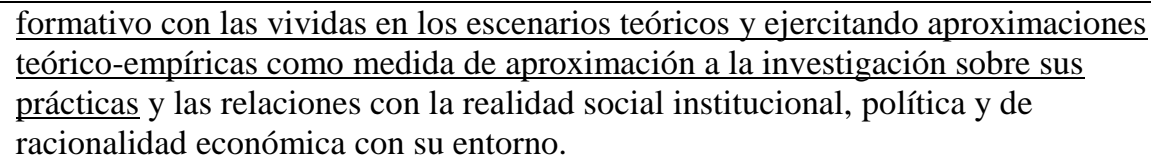

\section{Conclusiones}

La participación del profesional de enfermería en la promoción de la salud y la prevención de la enfermedad, utilizando como herramienta la vigilancia epidemiológica o el monitoreo estratégico que brinda la epidemiología social, debe garantizar una visión integrada de los componentes en la promoción y prevención de las adicciones que, desde la profesión, se incorporan en el análisis y solución de una problemática como ésta. Así, en la utilización del proceso de enfermería al estudiar la etiología de la dependencia de drogas es importante tener en cuenta los factores no farmacológicos, como la disponibilidad, las modas, la presión del grupo y el aprendizaje previo con sustancias toleradas socialmente pero no exentas de riesgo. Además, considerar que la vulnerabilidad a la farmacodependencia está también relacionada con algunas características del individuo habiéndose incluso reconocido en algunos casos la existencia de factores hereditarios.

En el origen y el mantenimiento de una farmacodependencia intervienen numerosas variables, por esta razón el estudio y entendimiento requiere de un enfoque multidisciplinario. Algunas variables que son dignas de destacar son las propiedades farmacológicas de las sustancias, los condicionantes fisiológicos y psicológicos del consumidor, así como diversos factores sociales y ambientales.

El énfasis, seguirá siendo la preeminencia de la educación sanitaria y estrategias de intervención para contribuir al proceso de reinserción social, de manera interdisciplinaria y en cooperación interinstitucional. Enfatizando, en la génesis y el mantenimiento de una adicción intervienen numerosas variables, por esta razón el estudio de este fenómeno requiere de un enfoque multidisciplinario. 


\section{Bibliografía}

ANUIES (2000). La Educación Superior en el Siglo XXI, líneas estratégicas de desarrollo. Una propuesta de ANUIES, México.

ANUIES (2000). Evaluación del desempeño del personal académico. Análisis y propuesta de metodología básica. México.

Alemán, Mario. et. al., (1997) El Modelo Curricular del ITQ. Revista TeQ. México, Instituto Tecnológico de Querétaro, número 13, año 7.

Alanís, Antonio (2001). El Saber hacer en la profesión docente. México, Trillas

Argudín Yolanda (2001). Educación basada en competencias. Revista Magistrales. MéxicoPuebla Universidad Iberoamericana, núm. 20.

Casarini Ratto, Martha (1999). Teoría y diseño Curricular. México, Trillas.

Fawcett J. (2005). Analysis and evaluation of contemporary nursing knowledge:Nursing models and theories (2nd ed.) Philadelphia, F. A. Davis.

Ferreiro Gravié Ramón (2007). Estrategias didácticas del Aprendizaje Cooperativo. México, Trillas.

Freire, Paulo (1984). Educación y Cambio. México, Siglo XXI.

Gadamer, H-G. (1997) Verdad y Método. España, Sígueme.

Gobierno Federal (2000). Plan Nacional de Desarrollo 2000-2006. México, Gobierno de la república.

González, Ma. Teresa (1997). Desarrollo Histórico de la Escuela de Enfermería. México, UAQ.

González Casanova, Pablo (2001). La universidad del siglo XXI. México, Paidós.

Gonczi, Andrew (2006). Competencia laboral y educación basada en normas de competencia. México, Limusa. 
Gottlieb, L. N.\&Feeley, N., Dalton, C. K. (2005) The collaborative partnership approach to care: A delicate balance, Toronto: Elsevier-Mosby,

Guyot, Violeta (2010) Repensando la educación en nuestros tiempos. Otras miradas, otras voces. Buenos Aires, Novedades Educativas.

INPRFM (2012). Encuesta Nacional de Adicciones 2011: Reporte de Alcohol. Primera Edición. Instituto Nacional de Psiquiatría Ramón de la Fuente Muñiz; Instituto Nacional de Salud Pública; Secretaría de Salud. México. http://encuestas.insp.mx/ena/ena2011/ENA2011_alcohol.pdf

Kemmis, S. (2003) El currículo: más allá de la teoría de la reproducción. España, Morata.

Kérouac, S., Pepin, J., Ducharme, F., et Major, F. (2003) La Pensée infirmière. Canadá, Beauchemin.

Larrosa, Jorge (2008) Pedagogía profana. Estudios sobre lenguaje, subjetividad y formación. Buenos Aires, Novedades Educativas.

Orozco Fuentes, Bertha et. al. (2012), Pensar lo educativo, tejidos conceptuales. México, Plaza y Valdés.

McKena H. (2010) Nursingtheories and models. London, Routledge.

Meleis, A.I. (2010) Transitions Theory: Middle range and situation specific theories and nursing research and practice. New York, Springer.

Morín, Edgar (2010) La mente bien ordenada. España, Seix Barral.

Morán Aguilar, Victoria; Mendoza Robles, Alba Lily (2003) Teorías y Modelos de Enfermería. México, Trillas.

Plan Institucional de Desarrollo de la Facultad de Enfermería 2000-2006.

Plan Institucional de Desarrollo de la Universidad Autónoma de Querétaro 2000-2010.

Puiggrós y Gómez Sollano (coords.) (2004) Alternativas pedagógicas, sujetos y prospectiva de la educación latinoamericana. Buenos Aires, Miño y Dávila. 
Quintar, Estela (2008) Conversaciones Didácticas. Argentina, EDUCO.

Siles José (2009) Historia de la Enfermería. España, Editorial Agua Clara.

Zapata, Jacqueline (2003) Poíesis educativa. México, Fundap Educación. 